Publ. RIMS Kyoto Univ. 46 (2010), 201208

DOI $10.2977 /$ PRIMS/6

\title{
Dominated Bilinear Forms and 2-homogeneous Polynomials
}

\author{
by \\ Geraldo Botelho, Daniel Pellegrino and Pilar Rueda
}

\begin{abstract}
The main goal of this note is to establish a connection between the cotype of the Banach space $X$ and the parameters $r$ for which every 2-homogeneous polynomial on $X$ is $r$ dominated. Let $\cot X$ be the infimum of the cotypes assumed by $X$ and $(\cot X)^{*}$ be its conjugate. The main result of this note asserts that if $\cot X>2$, then for every $1 \leq r<(\cot X)^{*}$ there exists a non- $r$-dominated 2-homogeneous polynomial on $X$.
\end{abstract}

2010 Mathematics Subject Classification: 46G25, 46B20, 46B28.

Keywords: $r$-dominated multilinear form, $r$-dominated homogeneous polynomial, absolutely $(p ; q)$-summing mapping, cotype.

\section{$\S 1$. Introduction}

The notion of $p$-dominated multilinear mappings and homogeneous polynomials between Banach spaces plays an important role in the nonlinear theory of absolutely summing operators. It was introduced by Pietsch [17] and has been investigated by several authors since then (see, e.g., [5, 6] and references therein).

Let $X$ be a Banach space and $m$ be a positive integer. A continuous $m$ linear form $A$ on $X^{m}$ is $r$-dominated if $\left(A\left(x_{j}^{1}, \ldots, x_{j}^{m}\right)\right)_{j=1}^{\infty} \in \ell_{r / m}$ whenever $\left(x_{j}^{1}\right)_{j=1}^{\infty}, \ldots,\left(x_{j}^{m}\right)_{j=1}^{\infty}$ are weakly $r$-summable in $X$. In a similar way, a scalarvalued $m$-homogeneous polynomial $P$ on $X$ is $r$-dominated if $\left(P\left(x_{j}\right)\right)_{j=1}^{\infty} \in \ell_{r / m}$ whenever $\left(x_{j}\right)_{j=1}^{\infty}$ is weakly $r$-summable in $X$.

Communicated by H. Okamoto. Received May 9, 2009. Revised July 29, 2009.

G. Botelho: Faculdade de Matemática, Universidade Federal de Uberlândia, 38.400-902 Uberlândia, Brazil;

e-mail: botelho@ufu.br

D. Pellegrino: Departamento de Matemática, Universidade Federal da Paraíba, 58.051-900 João Pessoa, Brazil;

e-mail: dmpellegrino@gmail.com

P. Rueda: Departamento de Análisis Matemático, Universidad de Valencia, 46100 Burjasot - Valencia, Spain;

e-mail: pilar.rueda@uv.es

(C) 2010 Research Institute for Mathematical Sciences, Kyoto University. All rights reserved. 
In [11, Lemma 5.4] it is proved that for every infinite-dimensional Banach space $X$, every $p \geq 1$ and every $m \geq 3$, there exists a continuous non- $p$-dominated $m$-linear form on $X^{m}$. For polynomials the question has recently been settled in [6], where it is proved that for every infinite-dimensional Banach space $X$, every $p \geq 1$ and every $m \geq 3$, there exists a continuous non- $p$-dominated scalarvalued $m$-homogeneous polynomial on $X$. So, coincidence situations can occur only for $m=2$. Sometimes it happens that every continuous bilinear form on $X^{2}$ is 2-dominated, for example if $X$ is either an $\mathcal{L}_{\infty}$-space, the $\operatorname{disc}$ algebra $\mathcal{A}$ or the Hardy space $H^{\infty}$ (see [4, Proposition 2.1]). In this case every continuous bilinear form on $X^{2}$ and every continuous scalar-valued 2-homogeneous polynomial on $X$ are $r$-dominated for every $r \geq 2$. But what about $r$-dominated bilinear forms and 2-homogeneous polynomials for $1 \leq r<2$ ?

Those spaces $X$ that enjoy the property that all bilinear forms on $X^{2}$ are 1-dominated are all of cotype 2 (Example 1). In Proposition 3.2 we see that having cotype $2+\varepsilon$ for every $\varepsilon>0$ is a necessary condition. So, for a space $X$ such that $\cot X>2$ it is natural to investigate how close $r$ can be to 1 with the property that every bilinear form on $X^{2}$ (or 2-homogeneous polynomial on $X$ ) is $r$-dominated. For bilinear forms it is not difficult to see (Proposition 3.3 that $(\cot X)^{*}$, the conjugate of the number $\cot X$, is the closest $r$ can be to 1 . As usual, for polynomials the situation is more delicate. In the main result of this paper, Theorem 3.2 , we prove that the estimate $(\cot X)^{*}$ holds for 2 -homogeneous polynomials as well. We also point out that this result is in a sense sharp.

\section{$\S 2$. Notation}

Throughout this paper, $n$ and $m$ are positive integers, and $X$ and $Y$ will stand for Banach spaces over $\mathbb{K}=\mathbb{R}$ or $\mathbb{C}$. The Banach spaces of all continuous $m$-linear mappings $A: X^{m} \rightarrow Y$ and continuous $m$-homogeneous polynomials $P: X \rightarrow Y$, endowed with the usual sup norms, are denoted by $\mathcal{L}\left({ }^{m} X ; Y\right)$ and $\mathcal{P}\left({ }^{m} X ; Y\right)$, respectively $(\mathcal{L}(X ; Y)$ if $m=1)$. When $m=1$ and $Y=\mathbb{K}$ we write $X^{*}$ to denote the topological dual of $X$. The closed unit ball of $X$ is represented by $B_{X}$. The notation $\cot X$ denotes the infimum of the cotypes assumed by $X$. The identity operator on $X$ is denoted by $\mathrm{id}_{X}$. For details on the theory of multilinear mappings and homogeneous polynomials between Banach spaces we refer to [10, 14].

Given $r \in[0, \infty)$, let $\ell_{r}(X)$ be the Banach $(r$-Banach if $0<r<1)$ space of all absolutely $r$-summable sequences $\left(x_{j}\right)_{j=1}^{\infty}$ in $X$ with the norm $\left\|\left(x_{j}\right)_{j=1}^{\infty}\right\|_{r}=$ $\left(\sum_{j=1}^{\infty}\left\|x_{j}\right\|^{r}\right)^{1 / r}$. We denote by $\ell_{r}^{w}(X)$ the Banach $(r$-Banach if $0<r<1)$ space of all weakly $r$-summable sequences $\left(x_{j}\right)_{j=1}^{\infty}$ in $X$ with the norm $\left\|\left(x_{j}\right)_{j=1}^{\infty}\right\|_{w, r}=$ $\sup _{\varphi \in B_{X^{*}}}\left\|\left(\varphi\left(x_{j}\right)\right)_{j=1}^{\infty}\right\|_{r}$. 
Let $p, q>0$. An $m$-linear mapping $A \in \mathcal{L}\left({ }^{m} X ; Y\right)$ is absolutely $(p ; q)$-summing if $\left(A\left(x_{j}^{1}, \ldots, x_{j}^{m}\right)\right)_{j=1}^{\infty} \in \ell_{p}(Y)$ whenever $\left(x_{j}^{1}\right)_{j=1}^{\infty}, \ldots,\left(x_{j}^{m}\right)_{j=1}^{\infty} \in \ell_{q}^{w}(X)$. It is wellknown that $A$ is absolutely $(p ; q)$-summing if and only if there is a constant $C \geq 0$ such that

$$
\left(\sum_{j=1}^{n}\left\|A\left(x_{j}^{1}, \ldots, x_{j}^{m}\right)\right\|^{p}\right)^{1 / p} \leq C \prod_{k=1}^{m}\left\|\left(x_{j}^{k}\right)_{j=1}^{n}\right\|_{w, q}
$$

for every positive integer $n$ and every $x_{1}^{k}, \ldots, x_{n}^{k} \in X, k=1, \ldots, m$. The infimum of such $C$ is denoted by $\|A\|_{\operatorname{as}(p ; q)}$. The space of all absolutely $(p ; q)$-summing $m$ linear mappings from $X^{m}$ to $Y$ is denoted by $\mathcal{L}_{\mathrm{as}(p ; q)}\left({ }^{m} X ; Y\right)$, and $\|\cdot\|_{\mathrm{as}(p ; q)}$ is a complete norm $(p$-norm if $p<1)$ on $\mathcal{L}_{\text {as }(p ; q)}\left({ }^{m} X ; Y\right)$.

An $m$-homogeneous polynomial $P \in \mathcal{P}\left({ }^{m} X ; Y\right)$ is absolutely $(p ; q)$-summing if the symmetric $m$-linear mapping associated to $P$ is absolutely $(p ; q)$-summing, or, equivalently, if $\left(P\left(x_{j}\right)\right)_{j=1}^{\infty} \in \ell_{p}(Y)$ whenever $\left(x_{j}\right)_{j=1}^{\infty} \in \ell_{q}^{w}(X)$. It is well-known that $P$ is absolutely $(p ; q)$-summing if and only if there is a constant $C \geq 0$ such that

$$
\left(\sum_{j=1}^{n}\left\|P\left(x_{j}\right)\right\|^{p}\right)^{1 / p} \leq C\left(\left\|\left(x_{j}\right)_{j=1}^{n}\right\|_{w, q}\right)^{m}
$$

for every positive integer $n$ and every $x_{1}, \ldots, x_{n} \in X$. The infimum of such $C$ is denoted by $\|P\|_{\text {as }(p ; q)}$. The space of all absolutely $(p ; q)$-summing $m$-homogeneous polynomials from $X$ to $Y$ is denoted by $\mathcal{P}_{\text {as }(p ; q)}\left({ }^{m} X ; Y\right)$, and $\|\cdot\|_{\text {as }(p ; q)}$ is a complete norm $(p$-norm if $p<1)$ on $\mathcal{P}_{\text {as }(p ; q)}\left({ }^{m} X ; Y\right)$.

An $m$-homogeneous polynomial $P \in \mathcal{P}\left({ }^{m} X ; Y\right)$ is said to be $r$-dominated if it is absolutely $(r / m ; r)$-summing. In this case we write $\mathcal{P}_{\mathrm{d}, r}\left({ }^{m} X ; Y\right)$ and $\|\cdot\|_{\mathrm{d}, r}$ instead of $\mathcal{P}_{\mathrm{as}(r / m ; r)}\left({ }^{m} X ; Y\right)$ and $\|\cdot\|_{\mathrm{as}(r / m ; r)}$. As usual we write $\mathcal{P}_{\mathrm{d}, r}\left({ }^{m} X\right)$ and $\mathcal{P}\left({ }^{m} X\right)$ when $Y=\mathbb{K}$. The definition (and notation) for $r$-dominated multilinear mappings is analogous (for the notation just replace $\mathcal{P}$ by $\mathcal{L}$ ). For details we refer to [2, 4, 11].

\section{$\S 3$. Results}

First we establish the existence of Banach spaces on which every bilinear form (hence every scalar-valued 2-homogeneous polynomial) is 1-dominated. By $X \tilde{\otimes}_{\pi} X$ and $X \tilde{\otimes}_{\varepsilon} X$ we mean the completions of the tensor product $X \otimes X$ with respect to the projective norm $\pi$ and the injective norm $\varepsilon$, respectively. For the basics on tensor norms we refer to [8, 19.

By $\Pi_{r}$ we denote the ideal of absolutely $r$-summing linear operators. The following well-known factorization theorem (see, e.g., [17, Theorem 14] or [2, Proposition 46(a)]) will be useful a couple of times. 
Lemma 3.1. $\left.\mathcal{L}_{\mathrm{d}, r}\left({ }^{m} X ; Y\right)=\mathcal{L} \circ\left(\Pi_{r},{ }^{m}{ }^{m}\right), \Pi_{r}\right)\left({ }^{m} X ; Y\right)$ and $\mathcal{P}_{\mathrm{d}, r}\left({ }^{m} X ; Y\right)=\mathcal{P} \circ$ $\Pi_{r}\left({ }^{m} X ; Y\right)$ for all positive integers $m$ and Banach spaces $X$ and $Y$.

Proposition 3.1. Let $X$ be a cotype 2 space. Then $X \tilde{\otimes}_{\pi} X=X \tilde{\otimes}_{\varepsilon} X$ if and only if $\mathcal{L}_{\mathrm{d}, 1}\left({ }^{2} X\right)=\mathcal{L}\left({ }^{2} X\right)$.

Proof. This result is contained, in essence, in [11. We give the details for the sake of completeness.

Assume that $X \tilde{\otimes}_{\pi} X=X \tilde{\otimes}_{\varepsilon} X$ and let $A \in \mathcal{L}\left({ }^{2} X\right)$. Denoting the linearization of $A$ by $A_{L}$ we have $A_{L} \in\left(X \tilde{\otimes}_{\pi} X\right)^{\prime}=\left(X \tilde{\otimes}_{\varepsilon} X\right)^{\prime}$. Regarding $X$ as a subspace of $C\left(B_{X^{\prime}}\right)$ and using that $\varepsilon$ respects the formation of subspaces, $A_{L}$ admits a continuous extension to $C\left(B_{X^{\prime}}\right) \tilde{\otimes}_{\varepsilon} C\left(B_{X^{\prime}}\right)$, hence to $C\left(B_{X^{\prime}}\right) \tilde{\otimes}_{\pi} C\left(B_{X^{\prime}}\right)$ because $\varepsilon \leq \pi$. As bilinear forms on $C(K)$-spaces are 2-dominated, the bilinear form associated to this extension is 2-dominated. But restrictions of 2-dominated bilinear forms are 2-dominated as well, so $A$ is 2-dominated. Since 2-summing operators on cotype 2 spaces are 1-summing [9, Corollary 11.16(a)], it follows that $\Pi_{1}(X ; Y)=\Pi_{2}(X ; Y)$ for every $Y$, so by Lemma 3.1 we have

$$
\mathcal{L}_{\mathrm{d}, 2}\left({ }^{2} X\right)=\mathcal{L} \circ\left(\Pi_{2}, \Pi_{2}\right)\left({ }^{2} X\right)=\mathcal{L} \circ\left(\Pi_{1}, \Pi_{1}\right)\left({ }^{2} X\right)=\mathcal{L}_{\mathrm{d}, 1}\left({ }^{2} X\right) .
$$

It follows that $A$ is 1-dominated.

Conversely, assume that $\mathcal{L}_{\mathrm{d}, 1}\left({ }^{2} X\right)=\mathcal{L}\left({ }^{2} X\right)$ and let $A \in \mathcal{L}\left({ }^{2} X\right)$. Since 1dominated bilinear forms are 2-dominated, it follows that $A$ is 2-dominated, hence extendible by [13, Theorem 23]. Adapting the proof of [7, Proposition 1.1] to bilinear forms we conclude that $A$ is integral. Now apply [8, Ex. 4.12] to get $X \tilde{\otimes}_{\pi} X=X \tilde{\otimes}_{\varepsilon} X$.

Example 1. Pisier [18] proved that every cotype 2 space $E$ embeds isometrically in a cotype 2 space $X$ such that $X \tilde{\otimes}_{\pi} X=X \tilde{\otimes}_{\varepsilon} X$. So for every such space $X$ we have $\mathcal{L}_{\mathrm{d}, 1}\left({ }^{2} X\right)=\mathcal{L}\left({ }^{2} X\right)$.

It is easy to see that $\cot X=2$ is a necessary condition for every bilinear form on $X$ to be 1-dominated:

Proposition 3.2. If $\mathcal{L}_{\mathrm{d}, 1}\left({ }^{2} X\right)=\mathcal{L}\left({ }^{2} X\right)$, then $\cot X=2$.

Proof. By [1, Lemma 3.4] every bounded linear operator from $X$ to $X^{\prime}$ is 1summing. So, from [12, Proposition 8.1(2)] we conclude that the identity operator on $X$ is $(2 ; 1)$-summing. It follows that $\cot X=2$ by [9, Theorem 14.5].

Let $X$ be such that $\cot X>2$. Since we cannot have $\mathcal{L}_{\mathrm{d}, 1}\left({ }^{2} X\right)=\mathcal{L}\left({ }^{2} X\right)$, for which $r>1$ is it possible to have $\mathcal{L}_{\mathrm{d}, r}\left({ }^{2} X\right)=\mathcal{L}\left({ }^{2} X\right)$ ? Or, at least, $\mathcal{P}_{\mathrm{d}, r}\left({ }^{2} X\right)=$ 
$\mathcal{P}\left({ }^{2} X\right)$ ? In other words, we seek estimates for the numbers

$$
\mathcal{L}_{X}:=\inf \left\{r: \mathcal{L}_{\mathrm{d}, r}\left({ }^{2} X\right)=\mathcal{L}\left({ }^{2} X\right)\right\} \quad \text { and } \quad \mathcal{P}_{X}:=\inf \left\{r: \mathcal{P}_{\mathrm{d}, r}\left({ }^{2} X\right)=\mathcal{P}\left({ }^{2} X\right)\right\} .
$$

It is not difficult to give a lower bound for $\mathcal{L}_{X}$. By $q^{*}$ we denote the conjugate exponent of $q>1$.

Proposition 3.3. If $\cot X>2$, then $\mathcal{L}_{X} \geq(\cot X)^{*}$.

Proof. By Proposition 3.2 we know that $\mathcal{L}_{\mathrm{d}, 1}\left({ }^{2} X\right) \neq \mathcal{L}\left({ }^{2} X\right)$. Using the equality $\Pi_{1}(X ; Y)=\Pi_{r}(X ; Y)$ whenever $1 \leq r<(\cot X)^{*}[9$, Corollary 11.16(b)] and Lemma 3.1, we find that

$$
\mathcal{L}_{\mathrm{d}, r}\left({ }^{2} X\right)=\mathcal{L} \circ\left(\Pi_{r}, \Pi_{r}\right)\left({ }^{2} X\right)=\mathcal{L} \circ\left(\Pi_{1}, \Pi_{1}\right)\left({ }^{2} X\right)=\mathcal{L}_{\mathrm{d}, 1}\left({ }^{2} X\right) \neq \mathcal{L}\left({ }^{2} X\right)
$$

for every $1 \leq r<(\cot X)^{*}$, so the result follows.

It is not clear at once that the same holds for polynomials. Here the situation is usually more delicate: for instance, in [6] one can find a non- $r$-dominated bilinear form whose associated 2-homogeneous polynomial happens to be $r$-dominated. However, we shall prove in Theorem 3.2 that again $\mathcal{P}_{X} \geq(\cot X)^{*}$.

The following proof extends an argument which was first used in this context in 15 .

Theorem 3.1. Let $m$ be an even positive integer and $X$ be an infinite-dimensional real Banach space. If $q<1$ and $\mathcal{P}_{\operatorname{as}(q ; r)}\left({ }^{m} X\right)=\mathcal{P}\left({ }^{m} X\right)$, then $\operatorname{id}_{X}$ is absolutely $\left(\frac{m q}{1-q}, r\right)$-summing.

Proof. The open mapping theorem gives us a constant $K>0$ such that $\|Q\|_{\text {as }(q ; r)}$ $\leq K\|Q\|$ for all continuous $m$-homogeneous polynomials $Q: X \rightarrow Y$.

Let $n \in \mathbb{N}$ and $x_{1}, \ldots, x_{n} \in X$ be given. Consider $x_{1}^{*}, \ldots, x_{n}^{*} \in B_{X^{*}}$ such that $x_{j}^{*}\left(x_{j}\right)=\left\|x_{j}\right\|$ for every $j=1, \ldots, n$. Let $\mu_{1}, \ldots, \mu_{n}$ be real numbers with $\sum_{j=1}^{n}\left|\mu_{j}\right|^{s}=1$, where $s=1 / q$. Define $P: X \rightarrow \mathbb{R}$ by

$$
P(x)=\sum_{j=1}^{n}\left|\mu_{j}\right|^{1 / q} x_{j}^{*}(x)^{m} \quad \text { for every } x \in X .
$$

Since $m$ is even and $\mathbb{K}=\mathbb{R}$, it follows that $P(x) \geq 0$ for every $x \in X$. Also, $|P(x)|=P(x) \geq\left|\mu_{k}\right|^{1 / q} x_{k}^{*}(x)^{m}$ for every $x \in X$ and every $k=1, \ldots, n$. From

$$
|P(x)|=\left.\left.\left|\sum_{j=1}^{n}\right| \mu_{j}\right|^{1 / q} x_{j}^{*}(x)^{m}\left|\leq\|x\|^{m} \sum_{j=1}^{n}\right| \mu_{j}\right|^{1 / q}=\|x\|^{m}
$$


we conclude that $\|P\|_{\text {as }(q ; r)} \leq K\|P\| \leq K$. So

$$
\begin{aligned}
\left(\sum_{j=1}^{n}\left\|x_{j}\right\|^{m q}\left|\mu_{j}\right|\right)^{1 / q} & =\left(\sum_{j=1}^{n}\left(\left\|x_{j}\right\|^{m}\left|\mu_{j}\right|^{1 / q}\right)^{q}\right)^{1 / q} \leq\left(\sum_{j=1}^{n} \mid P\left(x_{j}\right)^{q}\right)^{1 / q} \\
& \leq\|P\|_{\operatorname{as}(q ; r)}\left(\left\|\left(x_{j}\right)_{j=1}^{n}\right\|_{w, r}\right)^{m} .
\end{aligned}
$$

Observing that this last inequality holds whenever $\sum_{j=1}^{n}\left|\mu_{j}\right|^{s}=1$ and that $\frac{1}{s}+$ $\frac{1}{s /(s-1)}=1$ we have

$$
\begin{aligned}
\left(\sum_{j=1}^{n}\left\|x_{j}\right\|^{\frac{s}{s-1} m q}\right)^{1 / \frac{s}{s-1}} & =\sup \left\{\left|\sum_{j=1}^{n} \mu_{j}\left\|x_{j}\right\|^{m q}\right|: \sum_{j=1}^{n}\left|\mu_{j}\right|^{s}=1\right\} \\
& \leq \sup \left\{\sum_{j=1}^{n}\left|\mu_{j}\right|\left\|x_{j}\right\|^{m q}: \sum_{j=1}^{n}\left|\mu_{j}\right|^{s}=1\right\} \\
& \leq\|P\|_{\mathrm{as}(q ; r)}^{q}\left(\left\|\left(x_{j}\right)_{j=1}^{n}\right\|_{w, r}\right)^{m q} \leq K^{q}\left(\left\|\left(x_{j}\right)_{j=1}^{n}\right\|_{w, r}\right)^{m q} .
\end{aligned}
$$

It follows that

$$
\left(\sum_{j=1}^{n}\left\|x_{j}\right\|^{\frac{s}{s-1} m q}\right)^{1 / \frac{s}{s-1} m q} \leq K^{1 / m}\left\|\left(x_{j}\right)_{j=1}^{n}\right\|_{w, r} .
$$

Since $\frac{s}{s-1} m q=\frac{m q}{1-q}, n$ and $x_{1}, \ldots, x_{n} \in X$ are arbitrary, we conclude that $\operatorname{id}_{X}$ is $\left(\frac{m q}{1-q} ; r\right)$-summing.

The following theorem holds for spaces over $\mathbb{K}=\mathbb{R}$ or $\mathbb{C}$ :

Theorem 3.2. If $\cot X=q>2$, then $\mathcal{P}_{\mathrm{d}, r}\left({ }^{2} X\right) \neq \mathcal{P}\left({ }^{2} X\right)$ for $1 \leq r<q^{*}$, where $q^{*}$ is the conjugate of $q$. In other words, $\mathcal{P}_{X} \geq q^{*}$.

Proof. Real case: Let $1 \leq r<q^{*}$. Combining Lemma 3.1 and [9, Corollary 11.16(b)] it is immediate that $\mathcal{P}_{\mathrm{d}, r}\left({ }^{2} X\right)=\mathcal{P}_{\mathrm{d}, 1}\left({ }^{2} X\right)$. If $\mathcal{P}_{\mathrm{d}, r}\left({ }^{2} X\right)=\mathcal{P}_{\mathrm{d}, 1}\left({ }^{2} X\right)=$ $\mathcal{P}\left({ }^{2} X\right)$, from Theorem 3.1 we could conclude that $\operatorname{id}_{X}$ is $(2 ; 1)$-summing, but this is impossible because $\cot X>2$.

Complex case: If $X$ is a complex Banach space, $\cot X=q>2$ and $1 \leq r<q^{*}$, then by [3, Lemma 3.1] we know that $\cot X_{\mathbb{R}}=q>2$, so there is a non- $r$-dominated polynomial $P \in \mathcal{P}\left({ }^{2} X_{\mathbb{R}}\right)$. Denoting by $\widetilde{P}$ the complexification of $P$ we see that $\widetilde{P} \in \mathcal{P}\left({ }^{2} X\right)$ and following the lines of [16, Proposition 4.30] it is not difficult to prove that $\widetilde{P}$ fails to be $r$-dominated either.

Remark. Let $X$ be any of the spaces constructed by Pisier [18. By Example 1 we know that $\mathcal{P}_{\mathrm{d}, 1}\left({ }^{2} X\right)=\mathcal{P}\left({ }^{2} X\right)$, which makes it clear that Theorem 3.2 is sharp in the sense that it is not valid for cotype 2 spaces. 
Conjecture. We conjecture that if $X$ is infinite-dimensional and $\mathcal{L}_{\mathrm{d}, 1}\left({ }^{2} X\right)=$ $\mathcal{L}\left({ }^{2} X\right)$, then $X \tilde{\otimes}_{\pi} X=X \tilde{\otimes}_{\varepsilon} X$. Observe that for an infinite-dimensional space $X$ with $\mathcal{L}_{\mathrm{d}, 1}\left({ }^{2} X\right)=\mathcal{L}\left({ }^{2} X\right)$ and $X \tilde{\otimes}_{\pi} X \neq X \tilde{\otimes}_{\varepsilon} X$, if any, we should have:

- $X$ has no unconditional basis [4, Theorem 3.2];

- $X$ has cotype $2+\varepsilon$ for every $\varepsilon>0$ (Proposition 3.2);

- $X$ does not have cotype 2 (Proposition 3.1);

- $X^{\prime}$ is a GT space [11, Theorem 3.4];

- every linear operator from $X$ to $X^{\prime}$ is absolutely 1-summing (by [1, Lemma 3.4] this is a consequence of $\left.\mathcal{L}_{\mathrm{d}, 1}\left({ }^{2} X\right)=\mathcal{L}\left({ }^{2} X\right)\right)$, in particular $X$ is Arens-regular;

- not every linear operator from $X$ to $X^{\prime}$ is integral (this is a consequence of $\left.X \tilde{\otimes}_{\pi} X \neq X \tilde{\otimes}_{\varepsilon} X\right)$.

\section{Acknowledgments}

Research of G. Botelho was supported by CNPq Grant 306981/2008-4.

Research of D. Pellegrino was supported by INCT-Matemática, CNPq Grants 620108/2008-8 (Ed. Casadinho) and 301237/2009-3.

Research of P. Rueda was supported by Ministerio de Ciencia e Innovación MTM2008-03211/MTM.

\section{References}

[1] G. Botelho, Cotype and absolutely summing multilinear mappings and homogeneous polynomials, Proc. Roy. Irish Acad. Sect. A 97 (1997), 145-153. Zbl 0903.46018 MR 1645283

[2] (2005/2006), 69-102. Zbl pre05058682 MR 2220454

[3] G. Botelho, H.-A. Braunss, H. Junek and D. Pellegrino, Inclusions and coincidences for multiple summing multilinear mappings, Proc. Amer. Math. Soc. 137 (2009), 991-1000. Zbl 1175.46037 MR 2457439

[4] G. Botelho and D. Pellegrino, Scalar-valued dominated polynomials on Banach spaces, Proc. Amer. Math. Soc. 134 (2006), 1743-1751. Zbl 1099.46033 MR 2204287

[5] G. Botelho, D. Pellegrino and P. Rueda, Pietsch's factorization theorem for dominated polynomials, J. Funct. Anal. 243 (2007), 257-269. Zbl 1118.46041 MR 2291438

[6] Dominated polynomials on infinite dimensional spaces, Proc. Amer. Math. Soc. 138 (2010), 209-216. Zbl pre05665568 MR 2550185

[7] D. Carando, Extendibility of polynomials and analytic functions on $\ell_{p}$, Studia Math. 145 (2001), 63-73. Zbl 0980.46034 MR 1828993

[8] A. Defant and K. Floret, Tensor norms and operator ideals, North-Holland Math. Stud. 176, North-Holland, 1993. Zbl 0774.46018 MR 1209438

[9] J. Diestel, H. Jarchow and A. Tonge, Absolutely summing operators, Cambridge Univ. Press, 1995. Zbl 0855.47016 MR 1342297

[10] S. Dineen, Complex analysis on infinite dimensional spaces, Springer, London, 1999. Zbl 1034.46504 MR 1705327 
[11] H. Jarchow, C. Palazuelos, D. Pérez-García and I. Villanueva, Hahn-Banach extension of multilinear forms and summability, J. Math. Anal. Appl. 336 (2007), 1161-1177. Zbl 1161.46025 MR 2353008

[12] J. Lindenstrauss and A. Pełczyński, Absolutely summing operators in $\mathcal{L}_{p}$ spaces and their applications, Studia Math. 29 (1968), 275-326. Zbl 0183.40501 MR 0231188

[13] Y. Meléndez and A. Tonge, Polynomials and the Pietsch Domination Theorem, Proc. Roy. Irish Acad Sect. A 99 (1999), 195-212. Zbl 0973.46037 MR 1881812

[14] J. Mujica, Complex analysis in Banach spaces, North-Holland Math. Stud. 120, NorthHolland, 1986. Zbl 0586.46040 MR 0842435

[15] D. Pellegrino, Cotype and absolutely summing homogeneous polynomials in $\mathcal{L}_{p}$ spaces, Studia Math. 157 (2003), 121-131. Z Zbl 1031.46052 MR 1980709

[16] D. Pérez-García, Operadores multilineales absolutamente sumantes, Thesis, Univ. Complutense de Madrid, 2003.

[17] A. Pietsch, Ideals of multilinear functionals (designs of theory), in: Proceedings of the second international conference on operator algebras, ideals and their applications in theoretical physics (Leipzig, 1989), Teubner-Texte Math. 67, Leipzig, 1984, 185-199. Zbl 0561.47037 MR 0763541

[18] G. Pisier, Counterexamples to a conjecture of Grothendieck, Acta Math. 151 (1983), 181208. Zbl 0542.46038 MR 0723009

[19] R. Ryan, Introduction to tensor products of Banach spaces, Springer, 2002. Zbl 1090.46001 MR 1888309 